tended converge over time. Antipsychotic dynamics are presented individually and by class, showing that Australia has a pattern of antipsychotic use that has no clear similarity to the US, UK or Asian practices.

Conclusion: Despite differences in prescribing earlier on, the convergence of prescribing patterns may indicate that there has been a diffusion of knowledge, perhaps based on CPGs, that has resulted in a common approach to antipsychotic use in the community.

\section{Antipsychotic prescribing trends in community treatment of schizophrenia: 1998-2003}

\author{
T Lambert ${ }^{1,2,3}$, B Singh $^{3}$ \\ ${ }^{1} \mathrm{OPEN} ;{ }^{2} \mathrm{ORYGEN}$ Research Centre; and ${ }^{3}$ The University of Melbourne, \\ Melbourne, Australia
}

Background: Following deinstitutionalization in Australia, there is little published literature on the use of antipsychotics in treating psychotic disorders under the new community-based paradigm of care. The present pharmacoepidemiological study was undertaken to examine the trends in antipsychotic prescribing in a number of service settings in Victoria from 1998 to 2003 to understand how such trends relate to community care.

Methods: Patients in community care teams from Northwestern Mental Health, Barwon Health, Grampians Health and Austin were sampled at various time points from 1998 (T1) to 2003 (T4) $(n=3966)$. Prescribing information and a slew of sociodemographic and adjunct treatment variables such as community treatment order status were ascertained.

Results: Between 1998 and 2003, prescriptions of second-generation ('atypical') antipsychotics grew to account for $78 \%$ of patients with schizophrenia being treated with these agents (RR 2.1, $P<0.01$ ). Firstgeneration ('typical') antipsychotics decreased to $<3 \%$ of prescriptions (RR .11, $P<0.01$ ) and depots fell and stabilized at about $30 \%$ of prescriptions (RR .64, $P<0.01)$.

Conclusions: Oral SGAs have largely replaced oral FGAs; yet, despite the apparent endorsement of the new agents, depot FGAs remain a mainstay of prescribing. This may reflect that up to $67 \%$ of patients show partial adherence with oral antipsychotics. The maintenance of depot prescribing meets the needs for prevention relapse as a primary outcome goal.

\section{Gender bias in the measurement of anxiety and depression}

\section{Leach}

Centre for Mental Health Research, Australian National University,

Canberra, Australia

Background: Epidemiological studies have consistently found that women are typically 1.5 to 3 times more likely to experience major depression and about twice as likely to experience an anxiety disorder. One possible contributor is item or gender bias in the diagnostic tools and continuous assessments used to measure these constructs. The present study examines whether symptoms assessed by the Goldberg Anxiety and Depression Scales have a gender bias.

Methods: A survey of 7485 people was carried out in the Canberra and surrounding Queanbeyan region (PATH Through Life Project). Confirmatory factor analysis was carried out to confirm the factor structure of the Goldberg scales and multiple group analysis was used investigate gender bias in the anxiety and depression items.

Results: The results showed that several items were significantly gender biased. However, as the effects were small, their impact is likely to be minimal.

Conclusions: It is important to investigate whether scales are gender biased when examining gender differences in anxiety and depression. This process helps to differentiate artifactual explanations from true differences.

\section{PsyCheck: responding to mental health issues within alcohol and drug treatment}

\author{
N Lee, J Cameron, T Brooke, S Roeg \\ Turning Point Drug and Alcohol Centre, Melbourne, Australia
}

Background: Integrated treatment is now the recommended practice for patients with comorbid mental health and alcohol and drug disorders. Up to $80 \%$ of patients present to alcohol and drug services with mental health problems, primarily the higher prevalence disorders (anxiety and depression). Alcohol and drug workers do not always have extensive mental health experience, and the focus of research and clinical programs is often on the more acute disorders, such as psychosis, meaning that few programs have been developed for this population. To provide truly integrated treatment, there is an urgent need to up-skill alcohol and drug workers to both screen and intervene with both clinical and subclinical mental health disorders. Aims/Methods: The PsyCheck Project has proceeded in three phases: 1) the development of screening and 
intervention tools, 2) the evaluation of the implementation of mental health screening and intervention within a range of drug and alcohol settings and 3) the best practice dissemination of the program. Each phase has undergone extensive evaluation. This presentation will overview the outcomes of phases 1 and 2 and outline the dissemination strategy including early evaluation results.

\section{Screening for distress including anxiety and depression in patients with cancer during in-patient admissions}

\section{S Lee', L Katona ${ }^{2}$, S de Bono², A de Castella'}

'Alfred Psychiatry Research Centre and Monash University; and ${ }^{2}$ The Alfred and Monash University, School of Psychology, Psychiatry \& Psychological Medicine, Melbourne, Australia

Background: Up to $60 \%$ of patients with cancer experience long-term distress, with $20 \%-35 \%$ experiencing anxiety or depression. Many distressed in-patients are not identified or referred for psychosocial support until in crisis. The aim of this project was to trial a new system of screening in-patients to better identify distressed patients, to examine the causes of their distress and to improve psychosocial referral.

Methods: A total of 115 in-patients (78 men, 38 women) on the oncology ward at the Alfred Hospital in Melbourne were screened using two self-report tools: 1) Brief Symptom Inventory-18 (BSI) assessing somatic distress, depression and anxiety and 2) distress thermometer (DT) assessing global distress $(0-10)$ and practical, emotional, spiritual and physical problems.

Results: About $52 \%$ of patients reported significant distress $(50 \%$ on the DT, $20 \%$ on the BSI). Of these, $25 \%$ had not been followed up by psychosocial services. Newly diagnosed patients reported greater distress than patients receiving palliative or curative treatment. A psychiatric history was also associated with significantly greater distress. Problems with fatigue $(66 \%)$, sleep $(50 \%)$, eating $(54 \%)$, fears $(41 \%)$, loss of interest (34\%), memory/concentration (31\%) and finances (20\%) were common. Family and friends, keeping busy, staying positive, staff expertise and care, setting goals, and maintaining a sense of normality were helpful coping strategies.

Conclusions: There is a high prevalence of unidentified distress in patients on the oncology ward, who would not normally be referred for psychosocial support. Proactive screening of patients can help detect distress and avoid crises by referral to psychosocial support services.

\section{NISAD's 'Gift of Hope' Brain Donor Program: a postmortem brain donor program for people with schizophrenia and allied disorders and those without mental illness}

\author{
Y Lim 1 , T Garrick², V Carr ${ }^{1,3}$, C Harper ${ }^{2}$ \\ 'Neuroscience Institute of Schizophrenia and Allied Disorders (NISAD); \\ 2Discipline of Pathology, The University of Sydney, Australia; and \\ ${ }^{3}$ Centre for Mental Health Studies, University of Newcastle, \\ Newcastle, Australia
}

Background: Research using human brain tissue has a vital role to play in increasing our understanding of the neurobiology of schizophrenia and related disorders. Such research has been limited by an inability to meet demands for well-characterized tissue from clinical populations. To help address this issue, ${ }^{1}$ Neuroscience Institute of Schizophrenia and Allied Disorders (NISAD) established the 'Gift of Hope' Brain Donor Program $(\mathrm{GoH})$. The program invites people to consent to donating their brain postmortem for medical research into neuropsychiatric disorders. At the time of death, NISAD staff coordinate and facilitate the donation. Australian and international research groups may then apply for access to use these stored tissues for research.

Methods: As donation for research purposes is a separate and specific consent from organ donation for transplantation, donors give consent to noncoronial autopsy and donation. A clinical interview using the Diagnostic Interview for Psychosis is conducted, lifestyle and medical information is collected and neuropsychological assessments are performed. Assessments are repeated at regular intervals to document changes in brain function. To ensure high-quality tissue for biomedical research, rapid notification protocols are in place to minimize postmortem delay. Projects are evaluated by a scientific advisory committee prior to allocation of tissues to researchers.

Results: The program has registered 378 expressions of interest and completed 143 enrollments, including 33 donors with schizophrenia. Since 1998, three donations have been successfully facilitated and incorporated into the NSW Tissue Resource Centre.

Conclusions: The GoH has received strong community support and will be critical to future schizophrenia research. 